\title{
3D-QSAR Studies of 2-Arylbenzoxazoles as Novel Cholesteryl Ester Transfer Protein Inhibitors
}

\author{
Jahan B. Ghasemi, ${ }^{*}$ Somayeh Pirhadi, and Mahnaz Ayati \\ Chemistry Department, Faculty of Sciences, K. N. Toosi University of Technology, Tehran, Iran \\ ${ }^{*}$ E-mail: jahan.ghasemi@gmail.com \\ Received August 23, 2010, Accepted December 20, 2010
}

\begin{abstract}
The 3D-QSAR study of 2-arylbenzoxazoles as novel cholesteryl ester transfer protein inhibitors was performed by comparative molecular field analysis (CoMFA), CoMFA region focusing (CoMFA-RF) for optimizing the region for the final PLS analysis, and comparative molecular similarity indices analysis (CoMSIA) methods to determine the factors required for the activity of these compounds. The best orientation was searched by all-orientation search strategy using AOS, to minimize the effect of the initial orientation of the structures. The predictive ability of CoMFARF and CoMSIA were determined using a test set of twelve compounds giving predictive correlation coefficients of 0.886 , and 0.754 respectively indicating good predictive power. Further, the robustness and sensitivity to chance correlation of the models were verified by bootstrapping and progressive scrambling analyses respectively. Based upon the information derived from CoMFA(RF) and CoMSIA, identified some key features that may be used to design new inhibitors for cholesteryl ester transfer protein.
\end{abstract}

Key Words: CoMFA, CoMFA region focusing, CoMSIA, Cholesteryl ester transfer protein inhibitors, All orientation search

\section{Introduction}

Atherosclerosis describes the principal progression in arterial dysfunction and remodeling that restricts blood flow to vessels in the peripheral vasculature and is ultimately manifested as coronary artery disease $(\mathrm{CAD})^{1-5}$ to date, the role of cholesteryl ester transfer protein (CETP) in coronary heart disease (CHD) is still not fully understood. Several epidemiological studies have demonstrated an inverse correlation between levels of high density lipoprotein (HDL) and the incidence of atherosclerotic cardiovascular disease i.e. plasma HDL is a negative risk factor for atherosclerosis. $\mathrm{CETP}^{6}$ is a hydrophobic glycoprotein which mediates the transfer and exchange of cholesteryl ester (CE) and triglycerides (TG) between plasma lipoproteins and plays an important role in HDL-CE and apolipoprotein a catabolism. It mediates transfer of neutral lipids between lipoproteins. ${ }^{7}$ CETP also promotes reverse cholestrol transport (RCT) by which peripheral cell cholestrol can be returned to liver for catabolism. Although the role of CETP in the development of atherosclerosis is controversial, CETP has been widely considered as a therapeutic target as human CETP deficiency result in markedly elevated HDL levels. Evidence exists that the consequences of CETP activity may depend on the metabolic setting, particularly on triglyceride levels. Accordingly, pharmacological CETP inhibition may reduce the risk of CAD in humans, but only in those with high triglyceride levels. ${ }^{8}$

The primary aim of 3D-QSAR methods is to establish a correlation of biological activities of a series of structurally and biologically characterized compounds with the spatial fingerprints of numerous field properties of each molecule, such as steric demand, lipophilicity, and electrostatic interactions. Typically, a 3D-QSAR analysis allows the identification of the pharmacophoric arrangement of molecular features in space and provides guidelines for the design of next-generation compounds with enhanced bioactivity or selectivity. The first applicable 3DQSAR method was proposed by Cramer et al. in $1988^{9}$. His program, CoMFA, was a major breakthrough in the field of 3D-QSAR.

The CoMFA approach describes the molecular properties by 3D steric (Lennard-Jones) and electrostatic (Coulomb) fields, evaluated over a lattice of points. Partial least-squares (PLS) method is used in order to correlate the variation of these properties with the variation of the biological response ${ }^{9}$. Unlike CoMFA, CoMSIA uses a Gaussian-type distance-dependent function to assess five fields of different physicochemical properties (i.e., steric, electrostatic, hydrophobic, and hydrogen bonding donor and acceptor). Similarly to the CoMFA, the fields are evaluated by PLS analysis. The advantage of CoMSIA over CoMFA method, is that no singularities occur at the atomic positions, since the fields are evaluated using a Gaussian function, and therefore, no arbitrary definitions of cutoff limits should be used. $^{10}$

Herein, we present a 3D-QSAR study to investigate the correlation of 2-arylbenzoxazoles derivatives with the inhibition of CETP by employing comparative molecular field analysis (CoMFA), and comparative molecular similarity indices analysis (CoMSIA).

\section{Materials and Methods}

Data Set. 3D QSAR studies were performed on a series of 50 2-arylbenzoxazoles as CETP inhibitors, to which the $\mathrm{IC}_{50}$ values were collected from published data. ${ }^{11}$ The activity $\left(\mathrm{IC}_{50}\right)$ values of some molecules were qualitatively (i.e. they have not exact quantities for activities) higher than 10 and some others higher than $32 \mu \mathrm{M}$, these molecules were removed from the data set. For the QSAR analysis the $\mathrm{IC}_{50}(\mu \mathrm{M})$ values were taken in molar range and were expressed in negative logarithmic 
Table 1. Structure of compounds

\begin{tabular}{|c|c|c|c|c|c|c|c|c|c|c|c|c|}
\hline \multirow{2}{*}{ Comp. } & \multirow[b]{2}{*}{$\mathrm{Z}$} & \multirow[b]{2}{*}{$\mathrm{pIC}_{50}$} & \multicolumn{5}{|c|}{$\bar{A}^{3}$} & \multicolumn{5}{|c|}{$2{ }^{3}$} \\
\hline & & & Comp. & 2 & 3 & 4 & $\mathrm{pIC}_{50}$ & Comp. & 2 & 3 & 4 & $\mathrm{pIC}_{50}$ \\
\hline $\mathrm{m} 1 *$ & $5-\mathrm{Me}$ & 5.959 & $\mathrm{~m} 21$ & & & & 6.155 & $\mathrm{~m} 45$ & $\mathrm{Me}$ & & $\mathrm{Cl}$ & 7.071 \\
\hline $\mathrm{m} 2$ & $5-\mathrm{OCF}_{3}$ & 5.032 & $\mathrm{~m} 22 *$ & $\mathrm{Me}$ & & & 6.553 & $\mathrm{~m} 46^{*}$ & & $\mathrm{CF}_{3}$ & $\mathrm{Cl}$ & 7.745 \\
\hline $\mathrm{m} 3$ & $5-\mathrm{SO}_{2} \mathrm{Et}$ & 4.959 & $\mathrm{~m} 23$ & & $\mathrm{Me}$ & & 6.161 & $\mathrm{~m} 47$ & & $\left.\mathrm{H}_{2}\right)_{3^{-}}$ & $\mathrm{Cl}$ & 7.824 \\
\hline $\mathrm{m} 4$ & $5-\mathrm{Ph}$ & 5.721 & $\mathrm{~m} 24$ & & & $\mathrm{Me}$ & 6.619 & $\mathrm{~m} 48^{*}$ & $\mathrm{Me}$ & & $\mathrm{Br}$ & 7.959 \\
\hline $\mathrm{m} 5$ & 5- $\mathrm{CH}_{2} \mathrm{OH}$ & 5.886 & $\mathrm{~m} 25$ & & $\mathrm{~F}$ & & 5.959 & $\mathrm{~m} 49$ & $\mathrm{Me}$ & COOMe & $\mathrm{Cl}$ & 7.745 \\
\hline $\mathrm{m} 6^{*}$ & 5-Ac & 5.921 & $\mathrm{~m} 26$ & $\mathrm{OMe}$ & & & 5.081 & $\mathrm{~m} 50$ & $\mathrm{Me}$ & $\mathrm{Me}$ & $\mathrm{Cl}$ & 8.000 \\
\hline $\mathrm{m} 7$ & $5-\mathrm{CF}_{3}$ & 6.229 & $\mathrm{~m} 27$ & & $\mathrm{Cl}$ & & 5.886 & & & & & \\
\hline $\mathrm{m} 8$ & $5-\mathrm{Cl}$ & 6.328 & M28* & & & $\mathrm{Cl}$ & 6.420 & & & & & \\
\hline $\mathrm{m} 9^{*}$ & $5-\mathrm{Br}$ & 6.357 & M29 & & i-Pr & & 6.046 & & & & & \\
\hline $\mathrm{m} 10$ & $5-\mathrm{NO}_{2}$ & 6.920 & $\mathrm{~m} 30$ & COON & & & 6.658 & & & & & \\
\hline $\mathrm{m} 11$ & $5-\mathrm{CN}$ & 7.244 & $\mathrm{~m} 31$ & $\mathrm{CF}_{3}$ & & & 5.721 & & & & & \\
\hline $\mathrm{m} 12$ & 6-Me & 4.887 & $\mathrm{~m} 32$ & & $\mathrm{Ph}$ & & 5.638 & & & & & \\
\hline $\mathrm{m} 13$ & 7-Me & 5.769 & $\mathrm{~m} 33$ & OCF & & & 6.699 & & & & & \\
\hline $\mathrm{m} 14$ & 5,7-DiMe & 6.553 & m34 & & $\mathrm{OCF}_{3}$ & & 5.444 & & & & & \\
\hline $\mathrm{m} 15$ & 5-Me-7-AC & 6.619 & $\mathrm{~m} 35^{*}$ & & $\mathrm{Cl}$ & $\mathrm{Cl}$ & 6.699 & & & & & \\
\hline $\mathrm{m} 16$ & $5-\mathrm{Cl}-7-\mathrm{Br}$ & 7.013 & $\mathrm{~m} 36$ & $\mathrm{Me}$ & & $\mathrm{Me}$ & 6.854 & & & & & \\
\hline $\mathrm{m} 17^{*}$ & 5,7-DiCl & 7.056 & $\mathrm{~m} 37$ & & $\mathrm{CF}_{3}$ & $\mathrm{Cl}$ & 6.921 & & & & & \\
\hline $\mathrm{m} 18$ & 5,7--BisCF 3 & 7.037 & $\mathrm{~m} 38^{*}$ & $\mathrm{Me}$ & & $\mathrm{Cl}$ & 7.018 & & & & & \\
\hline $\mathrm{m} 19^{*}$ & 5-CN-7-Cl & 7.284 & m39 & $\mathrm{Me}$ & & $\mathrm{Br}$ & 7.509 & & & & & \\
\hline \multirow[t]{5}{*}{$\mathrm{m} 20$} & $5-\mathrm{Cl}-7-\mathrm{NO}_{2}$ & 7.309 & $\mathrm{~m} 40$ & $\mathrm{Me}$ & & $\mathrm{F}$ & 7.638 & & & & & \\
\hline & & & $\mathrm{m} 41$ & & $\left.\mathrm{I}_{2}\right)_{3^{-}}$ & $\mathrm{Me}$ & 7.071 & & & & & \\
\hline & & & $\mathrm{m} 42 *$ & & $\left.\mathrm{I}_{2}\right)_{3^{-}}$ & $\mathrm{Cl}$ & 7.252 & & & & & \\
\hline & & & $\mathrm{m} 43$ & $\mathrm{Me}$ & COOMe & $\mathrm{Cl}$ & 7.377 & & & & & \\
\hline & & & $\mathrm{m} 44$ & $\mathrm{Me}$ & $\mathrm{Me}$ & $\mathrm{Cl}$ & 7.398 & & & & & \\
\hline
\end{tabular}

*prediction set.

units, $\mathrm{pIC}_{50}\left(-\log \mathrm{IC}_{50}\right)$. The chemical structures and corresponding $\mathrm{pIC}_{50}$ are listed in Table 1.

Molecular Modeling and Alignment. The QSAR modeling analysis, calculations, and visualizations were performed using the SYBYL 7.3 molecular modeling package (Tripose Inc., St. Louis, USA) running on a Red Hat Linux workstation 4.7. The $3 \mathrm{D}$ structures of the 2-arylbenzoxazole derivatives were constructed using the standard tools available in the SYBYL package. Energy minimization was performed using the Tripos force field with a distance dependent dielectric and the Powell conjugate gradient algorithm with a convergence criterion of 0.01 $\mathrm{kcal} / \mathrm{mol} \AA$. Partial atomic charges were calculated using the Gasteiger-Hückel method.

The set of inhibitors was divided in to a training and test set. The test set compounds were selected by considering both the distribution of biological data and structural diversity (Table 1). It can be seen that except the highest and lowest-activity compounds were included in the training set, the activity of test set is similar to that of the training set. 38 of inhibitors were used as the training set and 12 for the test set.

The accuracy of the prediction of CoMFA and CoMSIA dependent strongly on the structural alignment of the molecules. In this study molecular alignment was performed with the Distill

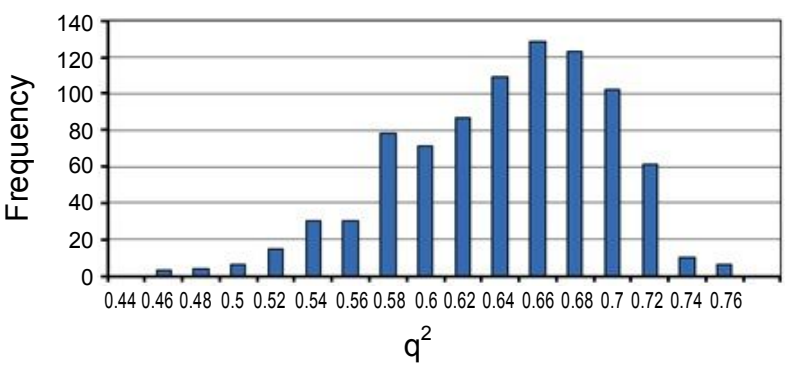

Figure 1. Frequency distribution of $\mathrm{q}^{2}$ values observed among all orientations for training set.

alignment method. The common structure and aligned compounds are displayed in Fig. 2(a) and (b), respectively. The lowest energy conformer of compound 50 was used as the template for superposition, assuming that it is the most active compound in the set and rest of the molecules were aligned on it. The common fragment produced by Distill program (without includeing bond types in rings) in SYBYL 7.3 was selected for rigid automatic alignment.

Since CoMFA models are highly sensitive to the different space orientations of the molecular aggregate with respect to 

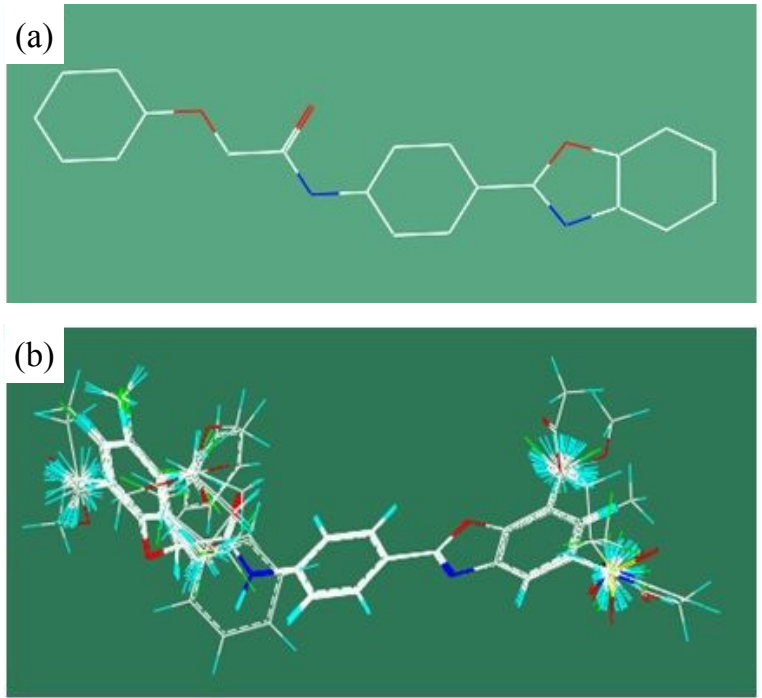

Figure 2. Common substructure in Distill alignment (a) and aligned compounds (b).

the lattice, for training set all-orientation search (AOS) was also conducted using the rotation procedure written in SYBYL programming language (SPL) ${ }^{12}$ (Fig. 1). For each orientation of the molecular aggregate, a conventional CoMFA was performed to obtain the optimal model.

CoMFA and CoMFA-RF. After consistently aligning the molecules within the lattice that extended $4 \AA$ units beyond the align molecules in all directions, a probe $\mathrm{sp}^{3}$ carbon atom with +1 charge was employed and steric and electrostatic interactions between the probe and molecules were calculated. Electrostatic interactions were modeled using a coulomb potential and van der Waals interactions using a Lennard-Jones potential. By sampling the steric and electrostatic fields surrounding a set of such molecules and correlated differences in these fields, a QSAR can be developed. To investigate the influence of different parameter settings on CoMFA, various steric and electrostatic cutoff and grid spacing values were also tried as suggested by Crammer et al ${ }^{9}$. Also important information could be lost when the grid spacing was too large or the probes were inadequately described.

CoMFA standard scaling applies the same weight to data from each lattice point in any given field. Region focusing is an iterative procedure which refines a model by increasing the weights for those lattice points which are most pertinent to the model. This enhances the resolution and predictive power $\left(\mathrm{q}^{2}\right.$; cross validated $\mathrm{r}^{2}$ ) of a subsequent PLS analysis. Technically, this corresponds to rotating the model components through a high-order space. ${ }^{13}$ PLS region focusing is intellectually analogous to the GOLPE approach and $\mathrm{q}^{2}$-GRS ${ }^{14,15}$

CoMSIA. In CoMSIA, the steric, electrostatic, hydrophobic, and hydrogen bond donor and acceptor descriptors were calculated at each lattice intersection of a regularly place grid of 1 and $2 \AA$. A probe atom with radius $1 \AA$, charge +1 , and hydrophobicity +1 was used to calculate the respective fields. The attenuation factor $(\alpha)$ was set to 0.3 . CoMSIA similarity indices $A_{F, k}^{q}(j)$ for a molecule $\mathrm{j}$ with atoms $\mathrm{i}$ at grid point $\mathrm{q}$ are calculated by Eq. 1

$$
A_{F, k}^{q}(j)=-\sum w_{\text {probe, }} w_{i k} e^{\alpha r_{i q}^{2}}
$$

in Eq. $1 \mathrm{~A}$ is the similarity index at grid point q, summed over all atoms $i$ of the molecule $j ; w_{\text {probe, }}$ is the probe atom with radius $1.0 \AA$, charge +1 , hydrophobicity +1 , hydrogen bond donating +1 , hydrogen bond accepting $+1 ; \mathrm{W}_{\mathrm{ik}}$ is the actual value of the physicochemical property $\mathrm{k}$ of atom $\mathrm{i} ; \mathrm{r}_{\mathrm{iq}}$ is the mutual distance between the probe atom at grid point $\mathrm{q}$ and atom $\mathrm{i}$ of the test molecule. A Gaussian type distance dependence function was used between the grid poin q and each atom i of the molecule.

Regression Analysis and Scrambling. To derive 3D QSAR models, the CoMFA and CoMSIA descriptors were used as independent variables and $\mathrm{pIC}_{50}$ activity value as a dependent variable. Partial least square (PLS) regression analysis was conducted in the SYBYL 7.3 package. The predictive ability of the models was evaluated by leave one out (LOO) cross-validation. The cross validated correlation coefficient, $\mathrm{q}^{2}$, was calculated using Eqs. 2 and 3.

$$
\begin{aligned}
& q^{2}=1-\frac{\text { PRESS }}{\sum_{i=1}^{N}\left(y_{i}-y_{m}\right)^{2}} \\
& \text { PRESS }=\sum_{i=1}^{N}\left(y_{\text {pred }_{i}}-y_{i}\right)^{2}
\end{aligned}
$$

where $y_{i}$ is the activity for training set compounds, $y_{m}$ is the mean observed value corresponding to the mean of the values for each cross validation group, and yPred, is the predicted activity for $y_{i}$.

The progressive scrambling method was carried out for the evaluation of the sensitivity of the 3D-QSAR model to chance correlations. The progressive scrambling analysis with the parameters; maximum: 8 bins, minimum: 2 bins and critical point: 0.85 were performed. Progressive scrambling with 2 - 6 components (latent variables) of the arylbenzoxazoles data produces three statistical data, the predictivity $\left(\mathrm{q}^{2}\right)$ of the model, the calculated cross-validated standard error (cSDEP) and the sensitivity to perturbation $\left(\mathrm{d}_{\mathrm{q}}{ }^{2} / \mathrm{dr}^{2} \mathrm{yy}^{\prime}\right)$.

As a final method of validation of the derived models, $\mathrm{pIC}_{50}$ of 12 compounds, as external test set, were predicted using models derived from the training set. The predictive ability of the models is expressed by the predictive $\mathrm{r}^{2}$ value and is calculated using the formula Eq. 4

$$
r_{\text {pred }}^{2}=\frac{S D-P R E S S}{S D}
$$

where SD is the sum of squared deviations between the biological activity of the test set and the mean activity of training set molecules, and PRESS is the sum of squared deviation between the actual and the predicted activities of the test set.

\section{Results}

CoMFA and CoMFA-RF Studies. The results of CoMFA studies are summarized in Table 2. The best model of all orientation search procedure was picked up, then the effect of 
Table 2. Statistical parameters of CoMFA with grid spacing land column filtering 1

\begin{tabular}{ccccccc}
\hline \multirow{2}{*}{$\begin{array}{c}\text { Statistical } \\
\text { parameters* }\end{array}$} & \multicolumn{6}{c}{ Cutoff value (kcal/mol) } \\
\cline { 2 - 7 } & 10 & 20 & 30 & 40 & 50 & 60 \\
\hline $\mathrm{q}^{2}$ & 0.569 & 0.745 & 0.713 & 0.693 & 0.693 & 0.668 \\
$\mathrm{R}_{\text {ncv }}$ & 0.919 & 0.905 & 0.936 & 0.932 & 0.932 & 0.923 \\
$\mathrm{~F}$ & 73.034 & 110.074 & 93.892 & 87.421 & 87.523 & 76.949 \\
$\mathrm{SEE}$ & 0.260 & 0.214 & 0.231 & 0.239 & 0.239 & 0.253 \\
$\mathrm{~S}$ & 0.576 & 0.531 & 0.558 & 0.575 & 0.586 & 0.591 \\
$\mathrm{E}$ & 0.424 & 0.469 & 0.442 & 0.425 & 0.414 & 0.409 \\
No. of LV & 5 & 5 & 5 & 5 & 5 & 5 \\
\hline
\end{tabular}

*statistical parameters have their original meanings.

Table 3. Model stability test for CoMFA model by progressive scrambling

\begin{tabular}{cccc}
\hline Components & $\mathrm{q}^{2}$ & cSDEP & $\mathrm{d}_{\mathrm{q}}^{2{ }^{\prime}} / \mathrm{dr}_{\mathrm{yy}}^{2}$ \\
\hline 2 & 0.241 & 0.762 & 0.825 \\
3 & 0.404 & 0.683 & 0.804 \\
4 & 0.465 & 0.656 & 0.773 \\
5 & 0.487 & 0.653 & 0.994 \\
6 & 0.458 & 0.685 & 1.827 \\
\hline
\end{tabular}

changing column filtering, cut off value, and grid spacing were investigated. The best results obtained at a column filtering of $1 \mathrm{kcal} / \mathrm{mol}$, cutoff value $20 \mathrm{kcal} / \mathrm{mol}$, and grid spacing 1 A for both steric and electrostatic fields. The optimal number of components was determined by selecting highest $\mathrm{q}^{2}$ value, which corresponds to lowest $S_{\text {press }}$ value. PLS analysis showed a high $\mathrm{q}^{2}$ value of 0.745 with five components for CoMFA. The non cross-validated PLS analysis results in a conventional $r^{2}$ of 0.905 , $F=110.074$, a standard error of estimation (SEE) of 0.214 . To assess the statistical confidence limits of the derived models, bootstrapping ${ }^{9}$ analysis was carried out with 100 runs. Bootstrapping involves the generation of many new dataset from that after randomly choosing samples from the original dataset. $\mathrm{A} \mathrm{r}_{\text {bs }}^{2}$ (average correlation coefficient for bootstrapping) of $0.964 \pm 0.011$ and a $\mathrm{SEE}_{\mathrm{bs}}$ (average standard error of estimate for bootstrapping) of $0.160 \pm 0.096$ suggested a good internal consistency of this model.

After focusing these fields, the $\mathrm{q}^{2}$ improved and produced highest $\mathrm{q}^{2}$ of 0.749 at column filtering $1 \mathrm{kcal} / \mathrm{mol}$ with five components, $\mathrm{F}=98.702, \mathrm{r}^{2}{ }_{\text {ncv }}=0.939, \mathrm{r}_{\text {pred }}^{2}=0.886$, a standard error of estimation of $0.226, \mathrm{r}^{2}$ bs of $0.975 \pm 0.004$, and a $\mathrm{SEE}_{\mathrm{bs}}$ of $0.143 \pm 0.088$. Steric field descriptors explain 0.568 of the variance, while the electrostatic descriptors explain 0.432 . The correlation between the predicted activities and the experimental activities are depicted in Fig. 3.

To evaluate of the sensitivity of the optimized CoMFA model to chance correlations, the leave-one-out (LOO) cross-validation and progressive scrambling analyses were performed by models having 2 - 6 components (latent variables). The complete details of the progressive scrambling can be find in the reference. ${ }^{16}$ In this approach, small random perturbations are introduced into a data set and the statistical results, the perturbation prediction

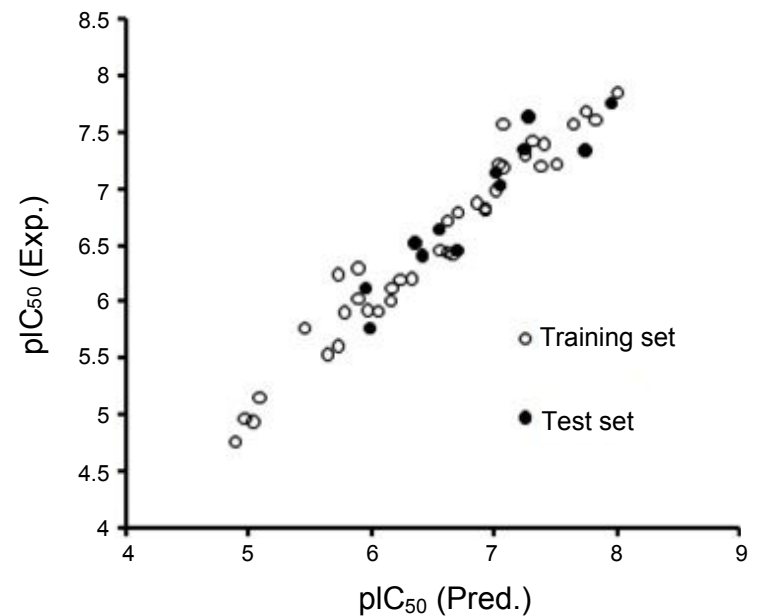

Figure 3. Plot of experimental against predicted activities for the training and test set compounds based on the best CoMFA-RF model.

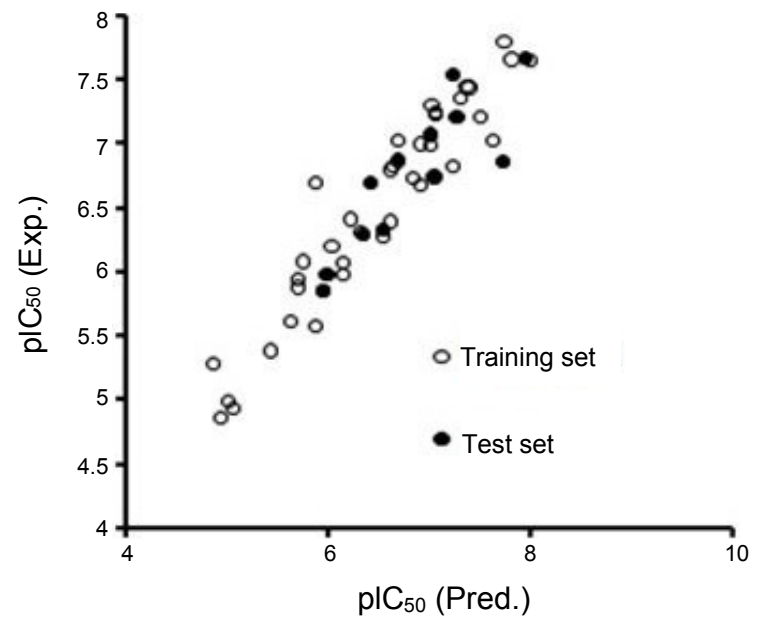

Figure 4. Plot of experimental against predicted activities for the training and test set compounds based on the best CoMSIA model.

$\left(\mathrm{q}^{2}\right)$, the calculated cross-validated standard error of prediction (cSDEP) as the function of the correlation coefficient between the true values (y) of the dependent variables and the perturbed values $\left(\mathrm{y}^{\prime}\right)$ of the dependent variables, and the slope of $\mathrm{q}^{2}$ (cross validated correlation coefficient) with respect correlation of the original dependent variables against the perturbed dependent variables $\left(\mathrm{d}_{\mathrm{q}}{ }^{2} / \mathrm{dr}^{2} \mathrm{yy}^{\prime}\right)$, are summarized in Table 3 . Specifically, in case of five components, the sensitivity to the perturbation $\mathrm{d}_{\mathrm{q}}{ }^{2} / \mathrm{dr}^{2} \mathrm{yy}^{\prime}=0.994$ and the prediction $\mathrm{q}^{2}=0.487$ produced by a progressive scrambling analyses were not dependent on chance correlation. Therefore, the data suggested that 5 components were optimal because a number of PLS components yielding $\mathrm{a} \mathrm{d}_{\mathrm{q}}{ }^{2} / \mathrm{dr}^{2}$ yy' $^{\prime}$ slope near unity should be optimal. ${ }^{17}$

CoMSIA Study. The CoMSIA analysis was done at a grid spacing 1 , and $2 \AA$, and the effect of column filtering was tested with the combination of five fields. The CoMSIA method defines explicit hydrophobic $(\mathrm{H})$ and hydrogen bond donor (D) and acceptor (A) descriptors in addition to the steric (S) and electrostatic (E) fields in CoMFA. To select the optimal result, we systematically changed the combination of fields. The high- 
est $\mathrm{q}^{2}$ of 0.539 was obtained with six components at a column filtering of $1 \mathrm{kcal} / \mathrm{mol}$, grid spacing of $1 \AA$, $\mathrm{F}=122.151$, noncross-validated $\mathrm{r}^{2}$ of $0.952, \mathrm{r}_{\text {pred }}^{2}=0.754, \mathrm{SEE}=0.187, \mathrm{r}_{\mathrm{bs}}^{2}=$ $0.928 \pm 0.03$, and $\mathrm{SEE}_{\mathrm{bs}}=0.238 \pm 0.15$ for the steric, electrostaic, hydrogen bond acceptor and hydrophobic fields. The corresponding field contributions of steric, electrostatic, hydrophobic, and hydrogen bond acceptor are $0.149,0.260,0.345$, and 0.246 respectively. The correlation between the predicted activities and the experimental activities are depicted in Fig. 4.

\section{Discussion}

CoMFA and CoMFA-RF Contour Maps. The CoMFA-RF's steric and electrostatic field contour plots obtained by Distill alignment are shown in Fig. 5. The steric interactions are represented by green- and yellow-colored contours, while electrostatic interactions are represented by red- and blue-colored contoures. In the green region of steric contour plot, bulky substitutes enhance biological activity, while in the yellow regions are likely to decrease the activity. Blue-colored contours represent regions where positive charge increases activity, whereas redcolored regions represent areas where negative charge enhances activity.

The blue region near $\mathrm{C} 5$ and $\mathrm{C} 7$ position of phenyl ring of benzoxazole indicates that biological activity will be decreased by electronegative group at the above- mentioned positions. Exactly, the inhibitory activity of compounds 9 (substituted by $\mathrm{Br}), 8(\mathrm{Cl}), 7\left(-\mathrm{CF}_{3}\right)$, and $2\left(-\mathrm{OCF}_{3}\right)$ are varied in order; $9>8>$ $7>2$. The biological activity of compound 18 with having electronegative substituent at this position is less than compounds 17,19 , and 20 , or compound 3 with more electronegative property shows a lower activity than compounds 5 and 6 .

The yellow region near the 5th and 6th substitution of phenyl ring of benzoxazole group suggests biological activity can be decreased by introduction of bulky groups at this position so the order of inhibitory activities of compounds $11,4,1$ is changed as; $11>1>4$. Also the sterically unfavored yellow region around $\mathrm{C} 5$ position of benzoxazole led to lower activity of compounds 2 - 6 in comparison compounds 22 - 24 .

Green and yellow regions are located at the 2 nd and $3 \mathrm{rd}$ position of phenyl ring (A), respectively. Sterically favorable green polyhedral indicating that substitution of hydrogen by bulkier group like $\left(-\mathrm{CF}_{3}\right)$ increase the activity, or compound 30 with bulky group at this position have higher activity than compound 22 . But the activity of compounds 23 and 25 is

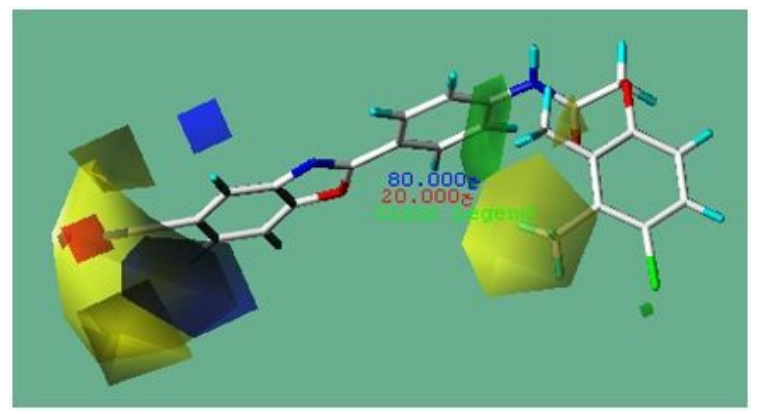

Figure 5. Contour maps of CoMFA-RF based on compound 50. higher than compounds 27, 32 and 34 or replacing bulky group $\left(-\mathrm{COOCH}_{3}\right)$ in compounds 43 and 49 with methyl group in compounds 50 and 44 can increase the activity, because yellow region surrounded the substitute at $\mathrm{C} 3$ of ring $\mathrm{A}$.

CoMSIA Contour Maps. The CoMSIA steric and electrosta-
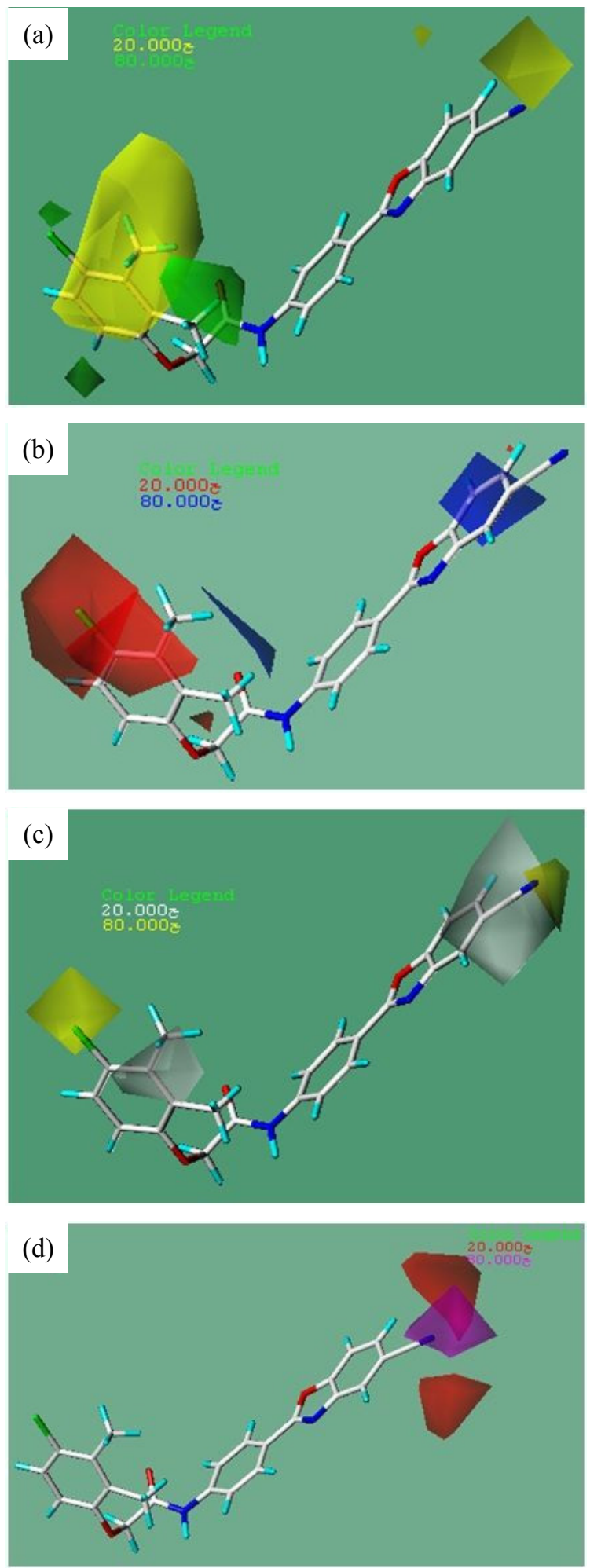

Figure 6. CoMSIA contour maps based on compound 50: steric (a), electrostatic (b), and hydrophobic (c), and Hydrogen bond acceptor (d) fields. 
tic field contour maps (Fig. 6(a) and (b)) are nearly similar to the corresponding CoMFA contour maps. In addition to CoMFA the appearance of red contour near $\mathrm{C} 3-4$ positions of ring A shows favor electronegative substituents and disfavor for electropositive substituents, for example $25(\mathrm{~F})>27(\mathrm{Cl}), 37\left(-\mathrm{CF}_{3}\right)$ $>35(\mathrm{Cl}), 40(\mathrm{~F})>39(\mathrm{Br}), 46\left(-\mathrm{CF}_{3}\right)>45(\mathrm{H}), 43(-\mathrm{COOMe})>$ $42\left(-\mathrm{CH}_{2}-\right)$, and $35(\mathrm{Cl})>28(\mathrm{H})$. Hydrophobicity is one of the most important properties related to biomolecular interactions. ${ }^{18}$ The term hydrophobicity refers to the force or corresponding energy that operates between two or more non polar solutes in water and arises from dispersive and electrostatic forces and the consequent entropic factor. A hydrophobic substance is soluble in non polar solvents but only sparingly soluble in water. In CoMSIA study, hydrophobic similarity index fields are constructed. The contour maps of hydrophobic and hydrogen bond acceptor fields are shown in Fig. 6(c) and (d). The yellow and white contours depict hydrophobic and hydrophilic favored regions, and magenta and red contours show favorable and unfavorable hydrogen bond acceptor groups, respectively. Compound 50 is served as reference molecule. The magenta contour near 5th substitution of phenyl ring of benzoxazole group suggests biological activity can be increased by introduction of hydrogen bond acceptor groups at this position, as a result the activity of compounds 10 and 11 with hydrogen bond acceptor groups such as $\mathrm{NO}_{2}, \mathrm{CN}$ is greater than 8 and 9 or the inhibitory activity of compounds $4-6$ are changed in the order; $6>5>4$. In compounds $45-50$, appearance of $\mathrm{CN}$ at this position led to higher activity, because these positions are surrounded by white region. Also there is a white region in 3rd substituent of ring A that the activity can be increased by introducing hydrophilic groups, for example $25>27$, and $46>$ 45 . The appearance a yellow region in $4^{\text {th }}$ position of this ring indicates why compound 39 is more potent than compound 38 .

\section{Conclusions}

CoMFA region focusing analysis using Distill alignment employing 12 molecules in test set provided the best model whereas the model with steric, electrostatic, hydrophobic, and hydrogen bond acceptor fields gave the best result in the case of CoMSIA analysis. To minimize the effect of the initial orientation of the structures, all-orientation search (AOS) was also conducted to CoMFA model using the rotation procedure. In general the CoMFA models are statistically superior to CoMSIA models. The CoMSIA steric and electrostatic field maps are in accordance with field distribution of CoMFA maps and consistent with structure-activity relationships. A high bootstrapped $\mathrm{r}^{2}$ values for CoMFA and CoMSIA models with small standard deviation assessed the statistical confidence limits and the robustness of the model. Also presence of chance correlation was checked with progressive scrambling. It has successfully identified the significance of various structural elements in 2-arylbenzoxazoles as novel cholesteryl ester transfer protein inhibitors possible to predict the ligand activities of newly designed inhibitors.

\section{References}

1. Wolfe, M. L.; Rader, D. J. Circulation 2004, 110, 1338.

2. Barter, P. J.; Brewer, H. B. J.; Chapman, M. J.; Hennekens, C. H.; Rader, D. J.; Tall, A. R. Arterioscler. Thromb. Vasc. Biol. 2003, 23,160 .

3. Ruggeri, R. B. Cur. Top. Med. Chem. 2005, 5, 257.

4. Cuchel, M.; Rader, D. J. J. Am. Coll. Cardiol. 2007, 50, 1956.

5. Parini, P.; Rudel, L. L. I. Arterioscler. Thromb. Vasc. Biol. 2003, 23,374

6. Huang, Z.; Inazo, A.; Mohara, A.; Higashikata, T.; Mabuchi, H. Clin. Sci. 2002, 103, 587.

7. De Grooth, G. J.; Kuivenhoven, J. A.; Stalenhoef, A. F.; de Graaf, J.; Zwinderman, A. H.; Posma, J. L.; Van Tol, A.; Kastelein, J. J. Circulation 2002, 105, 2159.

8. Boekholdt, S. M.; Kuivenhoven, J. A.; Wareham, N. J.; Peters, R. J. G.; Jukema, J. W.; Luben, R.; Bingham, S. A.; Day, N. E.; Kastelein, J. J. P.; Khaw, K. T. Circulation 2004, 110, 1418.

9. Cramer, R. D., III.; Patterson, D. E.; Bunce, J. D. J. Am. Chem. Soc. 1988, 110, 5959.

10. Klebe, G.; Abraham, U.; Meitzner, T. J. Med. Chem. 1994, 37, 4130.

11. Harikrishnan, L. S.; Kamau, M. G.; Herpin, T. F.; Morton, G. C.; Liu, Y.; Cooper, C. B.; Salvati, M. E.; Qiao, J. X.; Wang, T. C.; Adam, L. P.; Taylor, D. S.; Chen, A. Y. A.; Yin, X.; Seethala, R.; Peterson, T. L.; Nirschl, D. S.; Miller, A. V.; Weigelt, C. A.; Appiah, K. K.; O'Connell, J. C.; Lawrence, R. M. Bioorg. Med. Chem. Lett. 2008, 18, 2640.

12. Wang, R. X.; Gao, Y.; Liu, L.; Lai, L. H. J. Mol. Model. 1998, 4, 276.

13. QSAR ${ }^{\mathrm{TM}}$ Manual, SYBYL 7.3, Tripos, St. Louis, MO.USA.

14. Baroni, M.; Costantino, G.; Cruciani, G.; Riganelli, D.; Valigi, R.; Clementi, S. Quant. Struct. Act. Relat. 1993, 12, 9.

15. Tropsha, A.; Cho, S. J. J. Med. Chem. 1995, 38, 1060.

16. Clark, R. D.; Fox, P. C. J. Comput-Aided. Mol. Des. 2004, 18, 563.

17. Clark, R. D. J. Comput-Aided. Mol . Des. 2003, 17, 265.

18. Baurin, N.; Vangrevelinghe, E.; Allory, L. M. J. Med. Chem. 2000, 43, 1109 . 